\title{
Fe-Mg interdiffusion in wadsleyite and ringwoodite: Implications for water content of the mantle transition zone
}

\author{
BAOHUA ZHANG ${ }^{1}$ \\ ${ }^{1}$ Key Laboratory of Geoscience Big Data and Deep Resource \\ of Zhejiang Province, School of Earth Sciences, Zhejiang \\ University, Hangzhou 310027, China
}

Wadsleyite and ringwoodite are the major components of the upper and lower mantle transition zone (MTZ), which are capable of incorporating up to approximately 3 wt. $\%$ of $\mathrm{H}_{2} \mathrm{O}$ within their crystal structures. Therefore, the MTZ is considered to be a large water reservoir in deep Earth. It has been demonstrated that the incorporation of even tens of wt. ppm water could significantly affect physical properties of ringwoodite including seismic wave velocity and electrical conductivity. Despite the fundamental importance of diffusion data, there have been very few studies of diffusion at pressures relevant to the deep interior of the Earth mainly due to experimental difficulties. The fundamental question is whether or not large-scale chemical heterogeneities can be homogenized by solid-state diffusion of the MTZ minerals. Another key issue is how much water in the mantle transition zone, which can be inferred from the perspective of diffusion?

In this study, $\mathrm{Fe}-\mathrm{Mg}$ interdiffusion in wadsleyite and ringwoodite, the main constituent minerals of the mantle transition zone, was investigated as a function of water content (up to $0.6 \mathrm{wt} . \% \mathrm{H}_{2} \mathrm{O}$ ) at pressures of 16 and $20 \mathrm{GPa}$ and temperatures of $1373-1773 \mathrm{~K}$ by the diffusion couple method. The dependence of $\mathrm{Fe}-\mathrm{Mg}$ interdiffusivity on $\mathrm{Fe}$ concentration was determined using a Boltzmann-Matano method. Our results indicate that water significantly enhances the rates of $\mathrm{Fe}-\mathrm{Mg}$ interdiffusion in wadsleyite $(r=0.54)$ compared to that in ringwoodite $(r=0.25)$. Although our calculated diffusion coefficients as a function of depth in the mantle reveals that the MTZ is a zone of the maximum mixing efficiency for $\mathrm{Fe}$ and $\mathrm{Mg}$ under hydrous condition, homogenization of chemical heterogeneity is very limited in the MTZ only through solid-state diffusion. Comparison of the present conductivity-depth profiles deduced from $\mathrm{Fe}-\mathrm{Mg}$ interdiffusion with those observed by electromagnetic induction studies suggests that $\sim 0.1-0.5$ wt. $\% \mathrm{H}_{2} \mathrm{O}$ is required to account for the high conductivity anomalies in the upper part of the MTZ, whereas release of supercritical fluids or occurrence of partial melting at the stagnant slab roof may be a good candidate to account for the high conductivity anomalies in the lower part of the MTZ. 